\title{
A synchrotron for neutral molecules
}

\author{
Cynthia E. Heiner ${ }^{a}$, Hendrick L. Bethlem ${ }^{b}$, and Gerard Meijer ${ }^{a}$ \\ ${ }^{a}$ Fritz-Haber-Institut der Max-Planck-Gesellschaft, Faradayweg 4-6, D-14195 Berlin, Germany \\ ${ }^{b}$ Laser Centre Vrije Universiteit, De Boelelaan 1081, 1081HV Amsterdam, The Netherlands
}

(Dated: March 6, 2009)

\begin{abstract}
Here we report on a synchrotron for neutral molecules that consists of two hexapole focusers bent into a torus. By switching the voltages applied to the two half-rings, polar molecules can be confined in tight packets for up to a second as they revolve around the ring. In this article, the operation and trapping parameters of the molecular synchrotron are discussed. We plan to use a molecular synchrotron in the future as an arena for low-energy collision experiments.
\end{abstract}

\section{INTRODUCTION}

When, in 1919, Rutherford succeeded in splitting a nitrogen nucleus by subjecting it to a bombardment of natural $\alpha$-particles with energies of several $\mathrm{MeV}$, he at the same time laid the foundations for future collision studies involving accelerated particles [1]. The fact that $\alpha$ particles were projected in all directions and with only a limited amount of energy and intensity prompted Rutherford to express his "ambition to have available for study a copious supply of atoms and electrons which have an individual energy far transcending that of the $\alpha$ and $\beta$ particles from radioactive bodies" in an address given to the Royal Society in 1927 [2]. This statement served to fuel the race of developing an experimental means for producing high speed particles in order to perform intimate nuclear collisions. A forerunner in this pursuit was Ernest Lawrence, who - inspired by an article reporting on a three-stage accelerator for sodium ions [3] - wondered if instead of building many acceleration stages in a line, one should re-use an acceleration field by circulating positive ions back through it with the help of a magnetic field. With this novel thought, the cyclotron was born [4]. The energy that could be achieved with a cyclotron was about $15 \mathrm{MeV}$. At higher energies, relativistic effects make the particles slip out of phase with the acceleration fields. This problem was solved by employing the principle of phase stability proposed independently by McMillan[5] and Veksler [6]. Phase stability ensures that the particles remain in phase with the accelerating fields, allowing for acceleration to, in principle, limitless energies. This device was christened a synchrotron, as the magnetic and electric fields are synchronized with the travelling particle beam. Today, synchrotrons can accelerate particles to $\mathrm{TeV}$ energies, and the world awaits the results of future high energy collision experiments.

Here, we report on a variation to the well-known synchrotron - a synchrotron that has been adapted for use with neutral molecules. In this synchrotron we exploit the interaction of polar molecules with electric fields. A polar molecule is a molecule that has an unequal distribution of charges over the molecule, i.e., one side of the molecule is more positively charged and one side is more negatively charged. When such a molecule is placed in an electric field, the positive side of the molecule experi- ences a force along the direction of the electric field, while the negative side of the molecule experiences a force opposite to the direction of the electric field. In a homogeneous electric field these two forces cancel and there is no net force on the molecule. In an inhomogeneous electric field, on the other hand, the two forces no longer cancel. The force in this case is given by: $\vec{F}=\mu_{\text {eff }} \vec{\nabla}|\vec{E}|$, with $\mu_{\text {eff }}$ being the effective dipole moment, which is given by the projection of the dipole moment on to the electric field. This formula can be compared with Coulomb's law, $\vec{F}=-q \vec{\nabla} V$, which describes the force on a charged particle in an electric field. Thus, we can manipulate polar molecules using electric field differences in the same way that charged particles are manipulated using potential differences. Note, however, that there are a few complications. First, the $\mu_{\text {eff }}$ depends on the quantum state, hence it is crucial that the molecules remain in a single quantum state throughout the experiment. Second, the $\mu_{\text {eff }}$ is a function of the electric field, which introduces aggravating non-linearities in the focusing forces. Last but not least, the force a polar molecule experiences in an inhomogeneous field is typically some eight to ten orders of magnitude weaker than the force an ion experiences in an electric field.

Nevertheless, over the last couple of years, our group has demonstrated neutral analogues to different types of linear accelerators [7], [8], a buncher [9], a storage ring [10], and traps for charged particles [11], [12]. More recently we have demonstrated a neutral analogue to a synchrotron [13] - arguably the most celebrated tool of charged particle physicists. Here we give a detailed account of this molecular synchrotron.

Molecular synchrotrons can, in principle, be used to perform all the experiments that are performed in traps for molecules; for instance, the increased interaction time is useful for lifetime measurements and precision tests of fundamental physical constants. The advantage of a synchrotron is its independent control over the ring segments. This allows for the optimization of segments for particular tasks. The capability to store multiple packets of molecules in the ring makes it possible to study molecules over extended times without making concessions to the repetition frequency at which the experiment runs, e.g., packets can be stored for seconds while injection and detection take place at a $10 \mathrm{~Hz}$ rate. Our 
primary motivation for performing research into a molecular synchrotron is for studying cold collisions, which will be the subject of the next section.

\section{COLLISION EXPERIMENTS}

Collisions between molecules have been accurately studied using molecular beams. Such beams are prepared in a (supersonic) gas expansion in which the final beam, extracted from the core, contains fast molecules moving in a near collision-free environment and concentrated to only a few internal quantum states. The velocity of the molecules is determined by the mass of the carrier ('seed') gas and by the temperature and pressure of the source [14], which is typically about $2000 \mathrm{~m} / \mathrm{s}$ for $\mathrm{He}$ and $330 \mathrm{~m} / \mathrm{s}$ for Xe at room temperature. The translational and rotational temperatures of these beams are on the order of Kelvins, with vibrational temperatures being slightly higher, at tens of Kelvins. By adding electrostatic hexapoles along the beamline, molecules in a specific quantum state can be focused while molecules in all other quantum states can be de-focused, see for instance [15], [16].

The improved control over the internal degrees of freedom and the transverse motion of the molecules has left the forward velocity of the beam as the least well-defined parameter. Since the 1980 's, crossed beam set-ups have been built where the crossing angle of the intersecting beams can be varied, i.e., the collision energy can be varied. Volumes of invaluable knowledge into molecular collisions at relatively high energies, i.e. between 50$500 \mathrm{meV}$ (400-4000 $\mathrm{cm}^{-1}$ ), have been obtained in this way [17], [18], [19], [20], [21]. However, since the angle of the intersecting beams cannot be varied to arbitrarily small values and the velocity spread in the beam limits the obtainable energy resolution, experiments in the interesting low-energy regime between $0-50 \mathrm{meV}$ (0$400 \mathrm{~cm}^{-1}$ ) have remained largely elusive. However, it is in this low temperature regime - where the kinetic energy of the molecules is comparable to the rotational energy level splittings in the collision complex - where unusual events can occur between colliding molecules that may result in a "new" molecular species or complex that would otherwise be impossible to form or observe. For instance, let us assume the situation where after two molecules collide, they begin to rotate due to their translational energies being transferred, via the anisotropy of the potential energy surface, into rotational energy. This exchange may leave the molecules with insufficient translational energy to overcome the Van der Waals attraction, which effectively binds the molecules transiently together. These long lived excitations of the collision complex appear as sharp resonances in graphs of the cross section as a function of the collision energy. The position and width of these resonances provide an extremely sensitive probe of the interaction potential energy surfaces [22], [23], [24]. This type of resonance is ubiquitous in cold moleculemolecule collisions due to the manifold of internal molec- ular states that can lead to an equally large number of resonant states. An interesting consequence of the low temperature regime is also that the collision energy becomes comparable to energy shifts due to external electric or magnetic fields. This provides a mechanism of external control over chemical reactions [25].

To measure low-energy collisions, molecules must first be cooled; note that laser cooling - so famously applied for cooling atoms to sub-Kelvin temperatures - cannot be used for cooling molecules, as the complexity of their energy level structure, with its multitudes of vibrational and rotational components, precludes a simple extension of laser cooling [26]. Cold molecules can instead be produced either by placing molecules in a cryostat [27], [28], or by decelerating molecules in an expanded beam using electric, magnetic, or optical fields [29]. Our group has concentrated its efforts on deceleration techniques for polar molecules using electric fields, which is accomplished in a so-called Stark decelerator. This approach takes advantage of an expansion's internal cooling, and then decelerates the already cold beam in the laboratory frame by exploiting the interaction of polar molecules with inhomogeneous electric fields in much the same way that a linear particle accelerator (LINAC) exploits the interaction of charged particles with magnetic and electric fields. As the deceleration process is quantum-state specific, the state purity of the bunches of slow molecules emerging from the decelerator is close to $100 \%$. The velocity of the molecules can be tuned over a wide range and with a very narrow velocity distribution of typically $1-10 \mathrm{~m} / \mathrm{s}$. A pioneering crossed-beam scattering experiment has been recently performed using a Stark decelerated beam of $\mathrm{OH}$ molecules and a beam of Xe atoms [30]. In this experiment, the Xe beam had an average velocity of $300 \mathrm{~m} / \mathrm{s}$ while the velocity of the $\mathrm{OH}$ radicals was varied from 33 to $700 \mathrm{~m} / \mathrm{s}$, resulting in a total center-of-mass collision energy of $50 \mathrm{~cm}^{-1}$ to $400 \mathrm{~cm}^{-1}$.

In the above experiment, both the lowest attainable energy and the energy resolution were limited by the properties of the xenon beam. One can improve upon this by using two decelerated beams, however, obtaining the required densities in the scattering region poses a major obstacle. A way to increase the number of collisions that will take place is to load the decelerated beams into a molecular synchrotron. As opposed to the crossed beam geometry, in which the particles encounter each other only once, in a synchrotron the particles meet many times. For instance, in a ring containing 20 packets of molecules revolving in opposite directions, a packet having completed 100 round trips will have had 4,000 encounters. If losses, due to, e.g., collisions with the background gas, are kept to a minimum, then the total number of collisions that will take place will increase with the same factor. The molecules that undergo elastic or inelastic collisions will be ejected from the ring, resulting in a decrease of the number of stored molecules that scales with the number of round trips. The signal after some storage time with and without counter-propagating 
FIG. 1: Setup of a two-piece synchrotron. The two halfrings, separated by $2 \mathrm{~mm}$ gaps, are shown. The hexapole ring radius $\left(R_{\text {ring }}\right)$ is $125 \mathrm{~mm}$. The inset shows a crosssection of a hexapole bend and the transverse confinement fields. The electrodes are placed around a circle with a radius of $R_{\text {hex }}=4 \mathrm{~mm}$, and each electrode has a radius of $2 \mathrm{~mm}$. For transverse confinement the voltages are applied as shown; this creates a zero electric field in the hexapole center and a quadratically increasing field radially outwards. The contours represent the absolute electric field. The position of the molecules is given with respect to the center of the hexapole by the radial coordinate $r$ and the vertical coordinate $y$.

packets can be compared as a function of collisional energy [31]. Also, changing the applied electric and/or magnetic fields at the points of intersection, allows for the influence of external fields on the cross-section to be studied. Primary molecules to be investigated in this manner are $\mathrm{ND}_{3}, \mathrm{NH}_{3}, \mathrm{OH}$, metastable $\mathrm{CO}\left(a^{3} \Pi\right)$, and metastable $\operatorname{NH}\left(a^{1} \Delta\right)$, molecules that have all been decelerated and trapped already.

\section{OPERATION PRINCIPLES OF THE MOLECULAR SYNCHROTRON}

Synchrotrons for charged particles are typically built out of many individual elements placed along a circular track. These elements, each separated by some free-flight distance, are each designed for performing one specific task, e.g., steering, focusing, accelerating, or bunching the particles. A scheme for building a synchrotron for molecules in a similar fashion has been proposed [32], however, the performance of such a design is seriously hindered by the non-linearities of the forces experienced by the molecules. Instead we have opted for a radically different approach, as proposed by Crompvoets et al. [33]. In this design, illustrated in Figure 1, the synchrotron consists of two bent hexapoles separated by a small gap. All the fields necessary for steering, focusing, and bunching the molecules are created by changing the voltages applied to the hexapoles. In Section III A we will first address the transverse confinement forces, and then in Section III B we will address the longitudinal confinement forces. Results of experiments performed using deuterated ammonia molecules, $\mathrm{ND}_{3}$ are presented in both sections.

\section{A. Transverse confinement of neutral molecules}

\section{(i) theory}

For transverse confinement, alternating positive and negative voltages are applied to the hexapole electrodes. This creates a zero electric field in the hexapole center and an increasing field radially outwards, as sketched in the inset of Figure 1. Close to the center, the electric field is cylindrically symmetric and is given by

$$
|\vec{E}(r)|=\frac{3 V}{2 R_{\text {hex }}^{3}} r^{2},
$$

where $R_{\text {hex }}$ is the inner radius of the hexapole and $r$ is the radial distance from the axis of the hexapole. Note that when the radius of the hexapole is much smaller than the curvature of the ring, $R_{\text {hex }} \ll R_{\text {ring }}$, the electric field is only slightly distorted as compared to a linear hexapole [33].

The potential energy of an ammonia molecule in the low-field-seeking component of the $|J, K\rangle=|1,1\rangle$ state in an electric field is given by

$$
W_{\text {Stark }}=\sqrt{\left(\frac{1}{2} W_{\mathrm{inv}}\right)^{2}+\left(\frac{1}{2} \mu|\vec{E}|\right)^{2}}-\frac{1}{2} W_{\mathrm{inv}}
$$

where $W_{\text {inv }}$ is the inversion splitting, which is equal to $0.053 \mathrm{~cm}^{-1}$ for $\mathrm{ND}_{3}$, and $\mu$ is the dipole moment, which is equal to 1.5 Debye [34]. For small electric fields the Stark shift is quadratic, while for large electric fields (above $10 \mathrm{kV} / \mathrm{cm}$ ) the Stark shift becomes linear with $|\vec{E}|$.

In the electric field of the hexapole, the ammonia molecules experience a force towards the center of the hexapole, which can be written as

$$
\vec{F}=-\frac{k r \hat{\mathbf{r}}}{\sqrt{1+\left(\frac{W_{\text {inv }}}{k r^{2}}\right)^{2}}},
$$

where $k$ is the harmonic force constant given by

$$
k=\mu \frac{3 V}{2 R_{\text {hex }}^{3}} .
$$

In a curved hexapole, the molecules also experience a centrifugal force that depends on their forward velocity. The molecules are located at the radial position where the sum of the electrostatic (centripetal) and centrifugal forces is zero. This can be expressed as

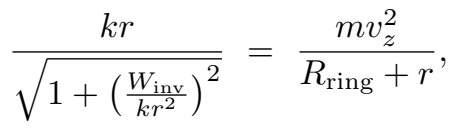

where $k$ is given by Equation 3.4 and $v_{z}$ is the forward velocity of the molecule in the lab-frame. Note that in the vertical $(\mathrm{y}-)$ direction, the molecules are located at the position where the sum of the electrostatic and the gravitational forces is zero; however the effect of gravity is negligible [33].

The particular radius where these two forces cancel is known as the equilibrium radius, $r_{\text {equi }}$. As a hypothetical molecule revolves around the ring at $r_{\text {equi }}$, it will form a closed orbit that remains at this radial position; this orbit is referred to as the equilibrium orbit. Molecules flying with the same forward velocity but with a different radial position or with a non-zero radial velocity will oscillate 
FIG. 2: The transverse trap depth as a function of the molecules's forward velocity is plotted both as the maximum transverse velocity accepted in the trap - the velocity spread is double this value - and in temperature units. The trap depth is shown for the perfectly linear case (solid line), assuming $\omega / 2 \pi=823 \mathrm{~Hz}$, and also for the actual case (crosses), including the effects of the inversion splitting.

around the equilibrium orbit with a frequency of around $800 \mathrm{~Hz}$; this is referred to as the betatron oscillation frequency. For an ideal case, an $\mathrm{ND}_{3}$ molecule circulating with a $v_{z}=87 \mathrm{~m} / \mathrm{s}$ would have a $r_{\text {equi }}$ of $2.30 \mathrm{~mm}$. However, when including $W_{\text {inv }}$ and field anharmonicites the $r_{\text {equi }}$ is $2.19 \mathrm{~mm}$. The $r_{\text {equi }}$ varies between $1 \mathrm{~mm}$ and $3.5 \mathrm{~mm}$ for velocities between $50-105 \mathrm{~m} / \mathrm{s}$. For velocities greater than $105 \mathrm{~m} / \mathrm{s}$, the $r_{\text {equi }}$ approaches the electrodes; molecules at this position will be lost from the ring.

The radial potential well that confines the molecules in a ring is the sum of the Stark energy of $\mathrm{ND}_{3}$ in the electric field of the hexapole and the pseudo-potential energy associated with the centrifugal force. The resulting potential is

$$
W(r)=\frac{1}{2} \frac{k r^{2}}{\sqrt{1+\left(\frac{W_{\text {inv }}}{k r^{2}}\right)^{2}}}-m v_{z}^{2} \ln \left|1+\frac{r}{R_{\text {ring }}}\right| .
$$

The potential well becomes less deep for molecules moving at increasing velocities. Assuming a linear force, the maximum radial position a molecule can have and still remain trapped is

$$
r=R_{\text {hex }}-r_{\text {equi }} .
$$

When $2 v_{z} \ll R_{\text {ring }} \omega, r_{\text {equi }}$ can be approximated by $v_{z}^{2} / R_{\text {ring }} \omega^{2}$, where $\omega$ is the angular trapping frequency. Hence the maximum transverse velocity a molecule can have and still remain trapped is

$$
v_{r}=\omega r \approx \omega\left(R_{\mathrm{hex}}-\frac{v_{z}^{2}}{R_{\mathrm{ring}} \omega^{2}}\right) .
$$

The relationship between the maximum transverse velocity and the forward velocity is shown in Figure 2 for both the ideal case (black), assuming a $\omega / 2 \pi=823 \mathrm{~Hz}$, and for the true case (crosses) including the nonlinearities in the potential. The vertical axis on the right plots the trap depth in terms of temperature units. The maximum transverse velocity that can be confined at $60 \mathrm{~m} / \mathrm{s}$ is $( \pm) 15 \mathrm{~m} / \mathrm{s}$, and is half that at $90 \mathrm{~m} / \mathrm{s}$ with $( \pm) 7.5 \mathrm{~m} / \mathrm{s}$; this is the velocity range for the synchrotron. This corresponds to a trap depth of about $200 \mathrm{mK}$ and $50 \mathrm{mK}$, respectively.

\section{(ii) experiment}

Our experimental set-up is schematically shown in Fig. 3. A pulsed beam of deuterated ammonia is formed by expanding a mixture of ca. $5 \% \mathrm{ND}_{3}$ in xenon into vacuum through a cooled solenoid valve. In the molecular beam, only the lowest rotational levels in the electronic and vibrational ground state of $\mathrm{ND}_{3}$ are occupied. The experiments are performed with molecules that reside in the low-field-seeking component of the $|\mathrm{J}, \mathrm{K}\rangle=|1,1\rangle$ state, which constitutes ca. $20 \%$ of all the $\mathrm{ND}_{3}$ molecules in the beam. A hexapole focuses the molecules into the 63-stage Stark decelerator, which decelerates them to velocities between $60 \mathrm{~m} / \mathrm{s}$ and $90 \mathrm{~m} / \mathrm{s}$. Two hexapoles and a buncher are used to focus the decelerated packet into the synchrotron. The combination of the decelerator and the focusing elements allows for complete control over the spatial and velocity distributions of the beam; further details of the injection beamline can be found elsewhere [35]. Once inside the synchrotron, the voltages are switched on to the confinement configuration. The $\mathrm{ND}_{3}$ molecules are detected at the injection point in the synchrotron using $(2+1)$-resonance enhanced multi-photon ionization (REMPI) with pulsed laser light around $317 \mathrm{~nm}$; immediately prior to firing the laser, the high voltages on the ring are switched off. The ions are extracted perpendicularly to the plane of the ring, and are then counted by an ion detector. The ion signal is proportional to the density of the ammonia molecules in the detection region of the ring.

To demonstrate the confinement and multi-loading of the synchrotron, we present measurements in Figure 4 of two packets of $\mathrm{ND}_{3}$ molecules stored in the synchrotron as a function of time after the ring is switched on $\left(t_{0}=0\right.$ for the first injection). Here a packet of $\mathrm{ND}_{3}$ molecules is decelerated to $92 \mathrm{~m} / \mathrm{s}$ in a Stark decelerator before being injected into the ring. The storage ring is switched on at $t_{0}=0$, and a second packet is injected at $t_{0}=100 \mathrm{~ms}$. Hence, the second packet trails the first by $35 \mathrm{~cm}$, or is roughly a half-ring behind. The peaks in the signal correspond to the packet(s) moving through the laser focus in the detection region upon completion of a round trip.

In the Figure, both the first and second packets are seen to spread out; the two dotted lines are $1 / t$ plots to guide the eye. A packet completes a round trip in $8.8 \mathrm{~ms}$. The first packet has a position spread of $12 \mathrm{~mm}$ after one round trip. After completing its $16^{\text {th }}$ round trip, the packet has broadened by an order of magnitude. The second packet shows a similar spreading trend. The time-integrated signal of the peaks, however, stays fairly constant. Thus, the molecules are not lost from the ring but rather spread out inside the ring. Similar measurements and conclusions were found using a dipole storage ring [10], [36], [33].

\section{B. Longitudinal confinement of neutral molecules}

\section{(i) theory}

This section describes the basic principles for implementing longitudinal (bunching) forces in the synchrotron to prevent the molecules from spreading out in 
FIG. 3: Experimental set-up of the injection beamline and molecular synchrotron. A pulsed beam of $\mathrm{ND}_{3}$ molecules is decelerated, bunched, and focused into the synchrotron. Molecules in the detection region are ionized using pulsed laser light around $317 \mathrm{~nm}$, extracted perpendicularly to the plane of the ring, and counted by an ion detector. The inset shows a photograph of the synchrotron.

FIG. 4: TOF profiles showing two packets of ammonia molecules, each moving at $v_{z}=92 \mathrm{~m} / \mathrm{s}$, revolving inside the hexapole storage ring; the two dotted lines are $1 / t$ plots to guide the eye. A packet of molecules takes $8.8 \mathrm{~ms}$ to complete one round trip and is estimated to have a (translational) temperature of $1.4 \mathrm{mK}$. The second packet is injected $100 \mathrm{~ms}$ after the first packet, and trails it by ca. $35 \mathrm{~cm}$ (almost $1 / 2$ the ring). Prior to injection of the second packet, the detection system is briefly switched off (indicated by the gray bar) to avoid saturation of the detector as the undecelerated part of the ammonia beam passes through the detection region. The inset shows a zoom-in of a TOF profile of the first packet after completing its $16^{\text {th }}-20^{\text {th }}$ round trips and the second packet after completing its $4^{\text {th }}-8^{\text {th }}$ round trips.

the synchrotron. Figure 5 depicts the buncher scheme. As molecules enter the gap from left to right, the voltages in bend 1 are turned off and the voltages in bend 2 are turned on to a bunching configuration [13]. The result is a smooth field gradient, which causes molecules in the low-field-seeking component of the $|J, K\rangle=|1,1\rangle$ state to gain Stark energy and consequently lose kinetic energy, i.e., the molecules are decelerated while they move into the gap. The Stark energy, or potential energy, in this configuration is denoted by $W_{1}$ in Figure 5 . As the molecules exit the gap, however, the voltages in bend 2 are turned off and the voltages in bend 1 are turned on to a bunching configuration. This creates the opposing field gradient, which causes molecules in a low-field-seeking state to lose Stark energy and thus gain kinetic energy, i.e., the molecules are accelerated as they move out of the gap. The potential energy in this configuration is denoted by $W_{2}$. The difference between $W_{1}$ and $W_{2}, \Delta W$, provides the restoring force in the longitudinal direction.

The time at which the two potentials are switched is defined with respect to the position (and velocity) of the so-called synchronous molecule - named such since the switching of the fields is synchronized to this particular molecule. This molecule is always at the same position in the gap when the fields are switched, $z^{\prime}$. In Figure 5(a), the buncher fields are switched after the synchronous molecule has passed the center of the gap, $z_{0}$, resulting in it spending more time on the decelerating slope of $W_{1}$ than on the accelerating slope of $W_{2}$, as marked in bold in the Figure. This leads to a net deceleration of the synchronous molecule, and, due to phase stability, of the whole packet of molecules. Likewise, as shown in Figure 5(b), if the buncher fields are switched before the synchronous molecule has reached the center of the gap, it will spend more time on the accelerating slope than on the decelerating slope, thus accelerating
FIG. 5: Principle for stably (a) decelerating, (b) accelerating, or (c) bunching molecules in the gaps of the ring. The potential energy curves $W_{1}$ and $W_{2}$, which a molecule flying at $r_{0}$ experiences in the vicinity of a gap, are plotted as a function of the $z$-position. The center of the gap, $z_{0}$, is denoted by the dotted line, and $z^{\prime}$ - the position at which the synchronous molecule is located when the fields are switched - is denoted by a dashed line. The bold sections in (a) and (b) denote the parts of the potential curves that are exploited for either deceleration or acceleration. Additionally in (c) the potential energy difference, $\Delta W$ is shown. A linear fit to the $\Delta W$ curve yields a slope, $2 W_{1}^{\prime}\left(z_{0}\right)$, of $0.065 \mathrm{~cm}^{-1} / \mathrm{mm}$ and approximates the linear restoring force, yielding an $\omega_{\text {syn }} / 2 \pi=46 \mathrm{~Hz}$.

the entire molecular packet.

It follows that simply bunching the molecules - that is re-focusing the molecules without altering their forward velocities - only occurs in the case that the fields are switched at $z^{\prime}=z_{0}$, i.e., where $\Delta W=0$. This is shown in Figure 5(c); operating the buncher in this way is referred to as normal operation. Here the potential energy curves $W_{1}$ and $W_{2}$ are shown along with the resulting $\Delta W$ curve for a molecule moving at a $r_{\text {equi }}=2.3 \mathrm{~mm}$. Looking at the $\Delta W$ curve, the only position at which the energy difference is zero is at $z_{0}-$ the position of the synchronous molecule under normal operation. The synchronous molecule will, therefore, lose and gain the same amount of kinetic energy per round trip. It follows that molecules in front of the synchronous molecule will lose more kinetic energy, and vice versa, molecules that are behind the synchronous molecule will gain more kinetic energy with respect to the synchronous molecule. Due to the mirror symmetry of the two potentials, $W_{1}\left(z_{0}-\Delta z\right)=W_{2}\left(z_{0}+\Delta z\right)$, thus allowing the difference between the two curves to be written as

$\Delta W=W_{1}(z+\Delta z)-W_{1}(z-\Delta z)=\left.2 \Delta z \frac{\partial W_{1}}{\partial z}\right|_{\mathrm{z}=\mathrm{z}_{0}}=2 W_{1}^{\prime}\left(z_{0}\right) \Delta z$.

We can introduce an average force, $\langle F\rangle$, which encompasses the force over one section of the ring with a length $L$ - consisting of one gap and one bend - around $z_{0}$ to be

$$
\langle F\rangle=-\frac{2 W_{1}^{\prime}\left(z_{0}\right) \cdot \Delta z}{L} ;
$$

if $\Delta z$ is small [37]. The $\langle F\rangle$ force is a linear restoring force, with an angular frequency, $\omega_{\text {syn }}$, that can be expressed by

$$
\omega_{\mathrm{syn}}=\sqrt{\frac{2 W_{1}^{\prime}\left(z_{0}\right)}{m L}} .
$$


FIG. 6: Round-trip TOF profiles [13]. The density of ammonia molecules at the detection zone inside the synchrotron is shown as a function of storage time. The two insets show zoom-ins of the TOF profiles of the $15^{\text {th }}$ and $40^{\text {th }}$ round trips. Their respective temporal widths, as determined from Gaussian fits, correspond to longitudinal position spreads of $4.8 \mathrm{~mm}$ and $3.0 \mathrm{~mm}$.

FIG. 7: TOF profiles of the density of ammonia molecules in the detection zone after completing 15 round trips with normal bunching and then five round trips with the buncher fields switched such that the energy in the packet was either (a) decreased or (b) increased between $0.015-0.06 \mathrm{~cm}^{-1}$ per gap, i.e. a total $\Delta E_{\text {kin }}=( \pm) 0.15-0.6 \mathrm{~cm}^{-1}$ (as labelled). The arrival-time of the upper bold trace $\left(\Delta E_{\text {kin }}=0 \mathrm{~cm}^{-1}\right)$ is assigned as $t_{0}$; this is the same TOF profile in both panels. The traces are vertically shifted for clarity, and the dashed lines are guides for the eye.

The value found for $2 W_{1}^{\prime}\left(z_{0}\right)$ from the linear fit of $\Delta W$ in Figure 5, through $z=0$, is $0.065 \mathrm{~cm}^{-1} / \mathrm{mm}$ for $\mathrm{ND}_{3}$, yielding an $\omega_{\text {syn }} / 2 \pi=46 \mathrm{~Hz}$. Taking the harmonic (linear) part of the buncher fields into consideration $\left(\Delta v=\omega_{\mathrm{syn}} \Delta z\right)$, molecules within a position interval of $\Delta z= \pm 2.5 \mathrm{~mm}$ and a velocity interval of $\Delta v_{z}= \pm 0.5 \mathrm{~m} / \mathrm{s}$ will experience a linear restoring force towards the synchronous molecule and oscillate around it. Thus the molecules are trapped in a travelling potential well that revolves around the ring.

\section{(ii) experiment}

For the experiments presented in this section, packets of ammonia molecules are decelerated to $87 \mathrm{~m} / \mathrm{s}$ and bunched in the synchrotron. As the molecules revolve around the ring, the voltages applied to the electrodes are switched between four configurations. While the molecules are in the bends, they are confined in two dimension by the confinement configuration, as described in the previous section. In the gap regions, the molecules are bunched (and accelerated or decelerated) in the manner just discussed. Note that the molecules are not transversely confined during the bunching process, thus they spread out in the two transverse directions during bunching. To offset this effect, the focusing force is increased by $30 \%$ during a short period of time before and after the molecules pass through the gap, by switching to a correcter configuration [13].

Figure 6 shows the time-of-flight (TOF) profile of the ammonia density in the detection zone of the ring as a function of storage time [13]. After an initial rapid decrease, and some modulations, the signal is seen to become relatively constant. Starting from about the $20^{\text {th }}$ round trip, the signal decays with a $1 / e$ time of 0.5 seconds, which is consistent with losses due to collisions with background gas in our $2 \times 10^{-8}$ mbar vacuum.

The TOF peaks are now much more narrow, approaching a delta-function on this scale, as compared to those in
Figure 4. To more clearly illustrate the absolute widths of these peaks, a zoom-in of two TOF profiles is shown as insets in the Figure. The TOF profiles for each of the 40 round trips shown in Figure 6 were fitted with Gaussians. From these fits, the longitudinal position spread can be inferred. The width of the bunched packet decreases from 8-6 $\mathrm{mm}$ in the first few round trips before stabilizing to around $3 \mathrm{~mm}$ at the $20^{\text {th }}$ round trip. This initial decrease is attributed to injected molecules that are at first accepted into the ring but possess trajectories that are unstable in the long term. As the width of the packet stays constant for the later round trips, one can conclude that the trajectories of these molecules are stable and they will, in principle, be confined indefinitely. Hence measurements into the ring dynamics were taken after the $15^{\text {th }}$ round trip, which is a compromise setting between good signal - as longer storage times suffer from losses due to background collisions - and studying the characteristics of the (truly) trapped molecules.

Measurements have been performed to experimentally map out the characteristics of the longitudinal potential well. Presented in Figure 7 is a series of TOF profiles in which ammonia molecules complete 15 round trips under normal bunching conditions and are then either (a) decelerated $\left(-\Delta E_{\text {kin }}\right)$ or (b) accelerated $\left(+\Delta E_{\text {kin }}\right)$ in each gap for the final five round trips. The upper bold trace in both graphs is the same TOF profile of the $20^{\text {th }}$ round trip with the ring operated normally $\left(\Delta E_{\text {kin }}=0\right)$. Here molecules are moving at $v_{z}=87 \mathrm{~m} / \mathrm{s}$, taking $9.3 \mathrm{~ms}$ to complete one round trip under normal bunching; their time-of-arrival after the completing the $20^{\text {th }}$ round trip $(186 \mathrm{~ms})$ is assigned to $t_{0}$. In the left panel, the traces from top to bottom have more energy removed from the packet, whereas in the right panel, the traces from top to bottom have more energy added to the packet. The total amount of energy extracted from/added to each packet is labelled in the center of the Figure, and ranges from $( \pm) 0.15$ to $( \pm) 0.60 \mathrm{~cm}^{-1}$.

Note that the more decelerated molecules are seen to arrive at earlier flight times (left panel), and viceversa, the more accelerated molecules are seen to arrive at later flight times (right panel). This may seem counterintuitive, since one would expect slower molecules to arrive in the detection zone later and faster molecules to arrive in the detection zone sooner, however, one simply needs to take the molecules' $z$-position in the gap into account. As seen in Figure 5, the molecules which are stably decelerated are actually those which are further ahead, due to the shifted $z^{\prime}$. Thus the $\mathrm{ND}_{3}$ density at different positive $z$-positions in the buncher can be determined by taking the difference between the timeof-arrival between the normally bunched molecules and those that were accelerated (decelerated), together with the final respective velocities of the packet. That is to say the TOF profiles in Figure $7(\mathbf{a})$ are probing the $\mathrm{ND}_{3}$ density at different positive $z$-positions in the buncher. In the same manner, the TOF profiles in Figure $7(\mathbf{b})$ are probing the $\mathrm{ND}_{3}$ density at different negative $z$-positions 
FIG. 8: Data analysis determined from the TOF profiles shown in Fig. 7. (a): The packet's total change in energy ( $\left.\Delta E_{\text {kin }}\right)$ is plotted as a function of their z-position in the gap with respect to the middle of the gap (plotted as circles $\bigcirc$ ). The $\Delta W$ curve (dashed) fits for a molecule with a $r_{\text {equi }}=1.5 \mathrm{~mm}$. The solid line shows a linear fit to the data. The slope of the fit, is $0.04 \mathrm{~cm}^{-1} / \mathrm{mm}$, and can be used in equation 3.11 to solve for the frequency of the buncher; in this case $\omega_{\text {syn }} / 2 \pi=39 \mathrm{~Hz}$. (b): The integrated signal of the stored molecules in the detection zone is plotted as a function of the packet's total change in energy $\left(\Delta E_{\mathrm{kin}}\right)$. The solid line shows a fit to the data using the equation Area $=\pi \cdot \omega_{\text {syn }}\left(z_{\max }-\left|a_{s} / \omega_{\text {syn }}^{2}\right|\right)^{2}$, where $z_{\text {max }}$ is the maximum distance at which a molecule can be away from $z_{0}$ and still be bunched, and $a_{s}$ is the longitudinal acceleration. This yields a $\omega_{\text {syn }} / 2 \pi=42 \mathrm{~Hz}$. (c): The position spread of a molecular packet that completes either four $(\triangle)$ or sixteen $(\bigcirc)$ round trips before being released from the longitudinal well is plotted as a function of expansion time; error bars are shown where necessary. The solid lines in the Figure show the results from $\Delta z(t)=\left(\left(\Delta z_{\mathrm{i}}\right)^{2}+\left(\Delta v_{\mathrm{z}} \cdot\left(t-t_{0}\right)\right)^{2}\right)^{1 / 2}$, where $t$ is the storage time and $t_{0}$ is the time at which the packet has its minimum position spread - the molecules are focused at the quarter-ring position by the last buncher forces before freely expanding. This yields a $\omega_{\text {syn }} / 2 \pi$ of $47 \mathrm{~Hz}$ and of $34 \mathrm{~Hz}$ for a packet released after completing its $4^{\text {th }}$ and $16^{\text {th }}$ round trip, respectively.

in the buncher, since the molecules that are stably accelerated are those which are further behind.

Based on these TOF profiles, one can determine the $\omega_{\text {syn }}$ in two ways. In Figure $8(\mathbf{a})$ the total change in energy $\left(\Delta E_{\text {kin }}\right)$ of the packet is plotted $(\bigcirc)$ as a function of their $z$-position in the gap, with respect to the middle of the gap. The molecules that are normally bunched arrive at $z=z_{0}\left(\Delta E_{\text {kin }}=0\right)$. A linear fit (solid line) to the data approximates the harmonic section of the buncher's restoring force. The slope of the fit, $0.04 \mathrm{~cm}^{-1} / \mathrm{mm}$, equals $2 W_{1}^{\prime}\left(z_{0}\right)$. Substituting this value into Equation 3.11 yields $\omega_{\text {syn }} / 2 \pi=39 \mathrm{~Hz}$. The $\Delta W$ curve shown in Figure $8(\mathbf{a})$ (dashed line) is for a $r_{\text {equi }}=1.5 \mathrm{~mm}$. This $\Delta W$ curve can be immediately compared to that in Figure $5(\mathbf{c})$.

In Figure 8(b) the integrated signal for each TOF profile shown in Figure 7 is plotted as a function of $\Delta E_{\text {kin }}$. The integrated signal is normalized to the trap's deepest point, $z=z_{0}\left(\Delta E_{\text {kin }}=0\right)$. The integrated signal becomes less as the trap becomes shallower towards the edges. The solid line in the figure shows the results from a fit to the data using a simple equation to determine the phase-space area (see caption of Figure 8). The synchrotron oscillation frequency is determined from this fit to be $42 \mathrm{~Hz}$

Measurements to probe the temperature in the longitudinal potential well are presented in Figure 8(c). Here the molecules are confined and bunched in normal operation until some storage time, at which point the buncher is turned off (confinement fields are still applied), and the molecules are released from the longitudinal well. The plot shows the position spread of the molecules as a function of expansion time - the colder the packet, the slower the expansion. Molecules completing their $4^{\text {th }}$ and $16^{\text {th }}$ round trips before being released are indicated by triangles $(\triangle)$ and circles $(\bigcirc)$, respectively. The solid lines in the Figure show the results from a simple fit to determine the position and velocity spreads. The position and velocity spreads of the packet released after the $4^{\text {th }}$ round trip are found to be $6 \mathrm{~mm}$ and $1.8 \mathrm{~m} / \mathrm{s}$, respectively. For the packet released after completing its $16^{\text {th }}$ round trip, a position and velocity spread of $4.8 \mathrm{~mm}$ and $1.0 \mathrm{~m} / \mathrm{s}$ is determined.
The velocity spread can be used to estimate a temperature of the packet [38]. For the packet released after 4 round trips the temperature is determined to be $1.4 \mathrm{mK}$, and for the packet released after 16 round trips the temperature is determined to be $0.5 \mathrm{mK}$. Note that additional measurements were taken for a packet released after completing its $25^{\text {th }}$ round trip, where the same temperature value of $0.5 \mathrm{mK}$ was determined. So as explained earlier, the hottest molecules, although initially confined, are slowly expelled from the ring. This is attributed to coupling between the longitudinal and transverse motions, which causes the initial decrease in the position spread observed in Figure 6 .

Additionally, one can use $\Delta z$ and $\Delta v_{z}$ to solve for $\omega_{\mathrm{syn}}$; this yields a synchrotron oscillation frequency of $47 \mathrm{~Hz}$ and $34 \mathrm{~Hz}$ for a packet released after completing its $4^{\text {th }}$ and $16^{\text {th }}$ round trip, respectively. The average value determined for $\omega_{\text {syn }} / 2 \pi$ is $40 \mathrm{~Hz}$. This value is in reasonable agreement with the calculations, which predict a value of $46 \mathrm{~Hz}$. Note that all three values determined experimentally for $\omega_{\text {syn }} / 2 \pi$ are based on measurements taken during a $50 \mathrm{~ms}$ interval. At a $40 \mathrm{~Hz}$ rate, the packet has then only completed two synchronous oscillations. Thus, it is not expected that one can assign a very precise frequency based on these measurements. In any case, some deviation from the theoretical value is expected due to misalignments in the ring.

\section{Coupling between the transverse and longitudinal motion}

Thus far, we have treated the transverse and longitudinal motion as being independent. In actual effect these motions are coupled. Coupling of the motion arises, for instance, because the cylindrical symmetry of the ring is broken by the inclusion of bunching elements. As a consequence, the confinement force varies as a function of the longitudinal position in the ring. For certain longitudinal velocities, the disturbance caused by the bunchers will add up, meaning that all trajectories become unstable and those particles will be lost. These unstable velocity regions are well known in charged par- 
FIG. 9: TOF profiles for the $99^{t h}, 100^{t h}$, and $101^{t h}$ round trips, in which the molecules are stored for ca. $1 \mathrm{~s}$. As determined from Gaussian fits to the profiles, the packet retains a temporal width of only $26.5 \mu \mathrm{s}$, corresponding to a position spread of $2.3 \mathrm{~mm}$, after flying a distance of over $80 \mathrm{~m}$.

ticle accelerator physics and great efforts are exerted in the design of a synchrotron to minimize these so-called "stopbands" or "motional resonances". These resonances can be understood and predicted from a simple model, and their strength can be reduced by implementing the correcter configuration before and after bunching in the synchrotron. Moreover, we have demonstrated elsewhere that these resonances can be completely avoided by appropriately changing the voltages applied to the ring electrodes for each desired velocity in the range between 60 and $90 \mathrm{~m} / \mathrm{s}$ [39]. Being able to tune the velocity of the stored beam is crucial for future collision studies.

Another coupling arises from the fact that the longitudinal force that the molecules experience depends on their radial position, $r$. This radial dependence also causes a coupling between the longitudinal and transverse motions of the molecules. However, due to the large difference in frequency between the synchrotron and betatron oscillations, this coupling is expected to be weak. For instance, a molecule moving at $87 \mathrm{~m} / \mathrm{s}$ has a synchrotron oscillation frequency of around $40 \mathrm{~Hz}$, whereas the betatron oscillation frequency is on the order of $800 \mathrm{~Hz}$.

\section{CONCLUSIONS AND OUTLOOK}

We have demonstrated a simple scheme for building a synchrotron for neutral molecules. We have characterized both the transverse and the longitudinal motion in the synchrotron, along with coupling effects between the two. This detailed knowledge allows us to store molecules for
100 round trips, or about $1 \mathrm{~s}$, as shown in Figure 9. In this measurement, the molecules have flown over $80 \mathrm{~m}$ and yet still remain in a tight packet with only a $2.3 \mathrm{~mm}$ length.

For future collision experiments, a synchrotron will be injected with molecules moving in opposite directions, so that the stored packets will meet as they revolve around the ring. Storing many such packets over an extended time increases the number of collisions by orders of magnitude. We have already presented the storing and bunching of two packets of molecules in the twopiece synchrotron elsewhere [13]; however, as the number of packets that can be stored in both directions is determined by the number of segments divided by two, clearly it is advantageous to divide the synchrotron into more segments. The number of collisions also scales as the number of packets squared. Moreover, the longitudinal acceptance scales inversely with the square root of the length of a single segment. By dividing the ring into many small segments one can approximate a ring using straight hexapoles instead of curved hexapole rods, which greatly simplifies the construction of the ring. Currently a new synchrotron consisting of 40 straight hexapoles is being tested at the Fritz-Haber-Institut. It is planned to use this device for future low-energy molecular collisions.

\section{ACKNOWLEDGEMENTS}

We gratefully acknowledge the contributions of many colleagues to the experiments described in this paper, in particular, David Carty, Floris Crompvoets, Henrik Haak, Georg Heyne, Jochen Küpper, Bas van de Meerakker, André van Roij, and Peter Zieger. H. L. B. acknowledges financial support from the Netherlands Organisation for Scientific Research (NWO) via a VIDIgrant, and the European Research Council via a Starting Grant.
[1] E. Rutherford, Science, 1919, 50, 467.

[2] E. Rutherford, Proc. Roy. Soc. A, 1928, 117, 300.

[3] R. Wideröe, Arch. f. Elektrot., 1928, 21, 387.

[4] E.O. Lawrence and M.S. Livingston, Phys. Rev., 1932, 40, 19.

[5] E.M. McMillan, Phys. Rev., 1945, 68, 143.

[6] V.I. Veksler, J. Phys. (USSR), 1945, 9, 153.

[7] H.L. Bethlem, G. Berden, and G. Meijer, Phys. Rev. Lett., 1999, 83, 1558.

[8] H.L. Bethlem, A.J.A. van Roij, R.T. Jongma, and G. Meijer, Phys. Rev. Lett., 2002, 88, 133003.

[9] F.M.H. Crompvoets, R.T. Jongma, H.L. Bethlem, A.J.A. van Roij, and G. Meijer, Phys. Rev. Lett., 2002, 89, 093004.

[10] F.M.H. Crompvoets, H.L. Bethlem, R.T. Jongma, and G. Meijer, Nature, 2001, 411, 174.
[11] H.L. Bethlem, G. Berden, F.M.H. Crompvoets, R.T. Jongma, A.J.A. van Roij, and G. Meijer, Nature, 2000, 406, 491.

[12] J. van Veldhoven, H.L. Bethlem, and G. Meijer, Phys. Rev. Lett., 2005, 94, 083001.

[13] C.E. Heiner, D. Carty, G. Meijer, and H.L. Bethlem Nature Phys., 2007, 3, 115.

[14] G. Scoles (editor), Atomic and molecular beam methods, 1988, vol. 1, Oxford University Press.

[15] J. Reuss, chap. in Atomic and molecular beam methods, 1988, vol. 1, Oxford University Press.

[16] S.R. Gandhi and R.B. Bernstein, J. Chem. Phys., 1987, 87, 6457.

[17] G.H. Hall, K. Liu, M.J. McAuliffe, C.F. Giese, and W.R. Gentry, J. Chem. Phys., 1984, 81, 5577.

[18] R.C. Macdonald and K. Liu, J. Chem. Phys., 1989, 91, 
821.

[19] D.M. Sonnenfroh, R.G. Macdonald, and K. Liu, J. Chem. Phys., 1991, 94, 6508.

[20] R. T. Skodje, D. Skouteris, D. E. Manolopoulos, S.-H. Lee, F. Dong, and K. Liu, Phys. Rev. Lett., 2000, 85, 1206.

[21] D. Skouteris, D.E. Manolopoulos, W. Bian, H.-J. Werner, L.-H. Lai, and K. Liu, Science, 1999, 286, 1713.

[22] N. Balakrishnan, A. Dalgarno, and R.C. Forrey, J. Chem. Phys., 2000, 113, 621.

[23] J.M. Hutson and P. Soldán, Int. Rev. Phys. Chem., 2007, 26, 1.

[24] E. Bodo and F.A. Gianturco, Int. Rev. Phys. Chem., 2006, 25, 313.

[25] R.V. Krems, Phys. Chem. Chem. Phys., 2008, 10, 4079.

[26] M.D. Di Rosa, Eur. Phys. J. D., 2004, 31, 395.

[27] J.M. Doyle, B. Friedrich, J. Kim, and D. Patterson, Phys. Rev. A, 1995, 52, R2515.

[28] J.D. Weinstein, R. deCarvalho, T. Guillet, B. Friedrich, and J.M. Doyle, Nature, 1998, 395, 148.

[29] S.Y.T. van de Meerakker, H.L. Bethlem, and G. Meijer, Nature Phys., 2008, 4, 595.

[30] J.J. Gilijamse, S. Hoekstra, S.Y.T. van de Meerakker, G.C. Groenenboom, and G. Meijer, Science, 2006, 313, 1617.

[31] Note that the detection sensitivity is less for measuring a loss signal, as in the case of the synchrotron, as opposed to a signal coming from the population of a new state. However, this can be (at least partly) compensated for by the fact that in a synchrotron elastic collisions contribute to the loss signal, and the elastic collision cross-sections are commonly larger than those for inelastic collisions.

[32] H. Nishimura, G. Lambertson, J.G. Kalnins, and H. Gould, Rev. Sci. Instrum., 2003, 74, 3271.

[33] F.M.H. Crompvoets, H.L. Bethlem, and G. Meijer, Adv. At. Mol. Opt. Phys. (eds. P.R. Berman and C.C. Lin) ISBN 0-12-003852-8, 2005, 52, 209-287.

[34] R.L. Bhattacharjee and L.H. Johnston and G.R. Sudhakaran and J.C. Sarker, J. Mol. Spec., 1989, 138, 38.

[35] C.E. Heiner, H.L. Bethlem, and G. Meijer, Phys. Chem. Chem. Phys., 2006, 8, 2666.

[36] F.M.H. Crompvoets, H.L. Bethlem, J. Küpper, A.J.A. van Roij, and G. Meijer, Phys. Rev. A, 2004, 69, 063406.

[37] Note that the notion of an average longitudinal force is only useful in the case when $\frac{L}{v_{z}} \ll \frac{1}{\omega_{\text {syn }}}$, i.e., when the molecules pass many gaps (bunchers) before completing one longitudinal oscillation. This condition is not strictly fulfilled in our case as the molecules pass only four gaps before completing an oscillation. This implies that the shape of the packet in phase-space changes as a function of the position in the ring; the position spread is larger at the gaps and smaller at the quarter-ring positions. Note that for molecules moving slower than $50 \mathrm{~m} / \mathrm{s}$ the model breaks down completely, and the buncher is no longer stable.

[38] M. Gupta and D. Herschbach, J. Phys. Chem. A, 1999, 103, 10670.

[39] C.E. Heiner, G. Meijer, and H.L. Bethlem Phys. Rev. A, 2008, 78, 030702(R). 